\title{
Elektrophorese von Enzymen auf Acetylcellulose-Streifen
}

\author{
Von \\ J. Methfessel \\ Aus dem Physiologisch-chemischen Institut der Martin-Lutber-Universität Halle/Saale (Direktor: Prof. Dr. H. Hanson)
}

(Der Schriftleitung zugegangen am 30. September 1963)

\begin{abstract}
In vergleichenden Versuchen an Amylase und B-Glucuronidase wird gezeigt, daß bei der Elektrophorese dieser Enzyme mit Acetylcellulose als Träger weit höhere Enzymaktivitäten erhalten bleiben als bei Papierelektrophorese.

Comparative studies on amylase and B-glucuronidase show, that in the electrophoresis of these enzymes much higher enzymic activities are preserved with acetyl-cellulose than with paper as the carrier.
\end{abstract}

Die Trägerelektrophorese von Enzymen ist heute eine viel angewandte Methodik zur Reindarstellung und Analyse dieser Wirkstoffe und neuerdings auch zur Differenzierung von Isoenzymen. Die Papierelektrophorese als das am wenigsten aufwendige Verfahren dieser Art ist jedoch mit z. T. ganz erheblichen Einbußen an Enzymaktivität verbunden (1-3). Diese Verluste sind neben Hitze-Inaktivierung unter anderem durch Kontakt mit Inhaltsstoffen des Papiers und durch Adsorption am Papier bedingt. Deshalb werden für enzymologische Untersuchungen als Trägermasse vielfach Schichten von Agar-Gel (4) oder Stärke-Gel (5) oder auch von synthetischen Polymerisationsprodukten bevorzugt, wobei gute Trennschärfe erreicht wird und die Enzymaktivität weitgehend erhalten bleibt. Kürzlich berichteten Dose und KRAUSE (G) über die besonderen Vorteile von „Sephadex“-Gel als Träger; diese Sub$\operatorname{stanz}$ ist in großer Reinheit erhältlich und gestattet es, alle elektrophoretisch behandelten Stöfe quantitativ $z u$ eluieren. Mit Rücksicht auf den größeren Aufwand, den die Gel-Elektrophorese jeglicher Art immerhin erfordert, halten wir es für angebracht, die guten Erfahrungen mitzuteilen, die wir bei der Elektrophorese von Enzymen mit Acetylcellulose-Streifen als Träger gemacht haben. Die hier mitgeteilten Versuche beschränken sich auf quantitative Untersuchungen über das Verhalten der Enzymaktivität von Amylase und $\beta$ Glucuronidase bei Elektrophorese auf Acetylcellulose ("Celluloseacetat") im Vergleich zur Papierelektrophorese.

\section{Methodik}

Amylase

\section{a) Enzympräparat}

Wir benutzten ein gereinigtes Trockenpräparat aus Malz, das überwiegend $\beta$-Amylase, zum kleineren Teil $\alpha$-Amylase enthielt. Wir bereiteten daraus eine etwa 10 proz. Lösung in 0,9 proz. $\mathrm{NaCl}$-Lösung, die $0,005 \mathrm{~m}$ in bezug auf $\mathrm{CaCl}_{2}$ war; sie wurde durch Zentrifugieren geklärt.

\section{b) Bebrütungsansätze}

Die Zusammensetzung des Enzym-Substrat-Gemisches war folgende:

$20 \mu l$ Amylaselösung

$4 \mathrm{ml} 0,2 \mathrm{~m}$ Acetatpuffer, $\mathrm{pH}=5,7$

$4 \mathrm{~m} l 2$ proz. Lösung von löslicher Stärke nach ZuLkowskI in Acetatpuffer.

Das Gemisch wurde $20 \mathrm{Min}$. bei $37^{\circ}$ inkubiert. In den Experimenten mit auf Träger aufgetragener Amylase wurde das Enzym zunächst 2 Stdn. mit dem Puffer (Volumenkorrektur bei feuchten Streifen!) unter mehrfachem Rühren des Ansatzes eluiert und dann mit der Stärkelösung inkubiert. $\mathrm{Zu}$ jeder Versuchsreihe wurde ein Kontrollansatz mit hitzedenaturiertem Enzym hergestellt.

\section{c) Auswertung}

Wir bedienten uns dazu der photometrischen Methode in Anlehnung an die Angaben von Noelting und Bernfeld (7): $2 \mathrm{ml}$ des filtrierten Reaktionsgemisches wurden zu $2 \mathrm{ml} 1$ proz. 3,5Dinitrosalicylsäurelösung in ein $25 \mathrm{~m} /$-Meßkölbchen gegeben, und dieses wurde $4 \mathrm{Min}$. in ein kochendes Wasserbad getaucht, dann rasch im Eisbad abgekühlt und mit Wasser zur Marke aufgefüllt. Photometrie der Rotbraunfärbung der Lösung im Pulfrich-Photometer (Grünfilter S 35, $1 \mathrm{~cm}$-Küvette).

\section{$\beta$-Glucuronidase}

\section{a) Enzympräparat.}

Wir gewannen ein hochwirksames Glucuronidasepräparat aus Rindermilz nach Fishman (8); es enthielt pro $\mathrm{m} / 85,0 \mathrm{mg}$ Protein, nach Weichselbaum (9) bestimmt.

\section{b) Bebrütungsansätze}

Zusammensetzung:

$50 \mu l$ Glucuronidaselösung (bzw. auf Trägerstreifen aufgetragenes Enzym)

$4 \mathrm{ml} 0,1 \mathrm{~m}$ Acetatpuffer; $\mathrm{pH}=5,0$

$4 \mathrm{~m} l$ 0,025 $m$ L-Menthyl- $\beta$-D-glucuronid, das biosynthetisch aus Kaninchenharn nach den Angaben von Qurck (10) gewonnen wurde.

Das Gemisch wurde $12 \mathrm{Stdn}$. bei $37^{\circ}$ inkubiert.

\section{c) Auswertung}

Die Substrathydrolyse wurde durch photometrische Bestimmung der freigesetzten Glucuronsäure verfolgt. Wir wählten dazu die 
Tab.1. Amylase-Aktivität vor und nach 71/2- und 15stdg. Elektrophorese auf Papier bzw. Acetylcellulose; $\left(110 \mathrm{~V} ; 20^{\circ} \mathrm{bzw} .2^{\circ}\right)$.

\begin{tabular}{|c|c|c|c|c|c|c|c|c|c|c|c|}
\hline \multirow{4}{*}{$\begin{array}{c}\text { Ansatz } \\
\text { Träger } \\
\text { Temp. }\left({ }^{\circ} \mathrm{C}\right)\end{array}$} & \multirow{4}{*}{$\begin{array}{c}\text { Enzym- } \\
\text { lösung } \\
\text { ohne } \\
20\end{array}$} & \multirow{2}{*}{\multicolumn{2}{|c|}{ Nach Auftragen }} & \multicolumn{8}{|c|}{ Nach Elektrophorese } \\
\hline & & & & \multicolumn{4}{|c|}{$71 / 2$ Stdn. } & \multicolumn{4}{|c|}{15 Stdn. } \\
\hline & & \multirow{2}{*}{$\begin{array}{c}\text { Papier } \\
20\end{array}$} & \multirow{2}{*}{$\begin{array}{l}\text { Acetyl- } \\
\text { cellulose } \\
20\end{array}$} & \multicolumn{2}{|c|}{ Papier } & \multicolumn{2}{|c|}{ Acetylcellulose } & \multicolumn{2}{|c|}{ Papier } & \multicolumn{2}{|c|}{ Acetylcellulose } \\
\hline & & & & 20 & 2 & 20 & 2 & 20 & 2 & 20 & 2 \\
\hline $\begin{array}{l}\text { Freigesetzte Maltose (mg) } \\
\text { Aktivität (\%) }\end{array}$ & $\begin{array}{c}2,646 \\
100\end{array}$ & $\begin{array}{l}1,610 \\
60,8\end{array}$ & $\begin{array}{l}2,273 \\
85,9\end{array}$ & $\begin{array}{l}0,648 \\
24,5\end{array}$ & $\begin{array}{c}1,447 \\
54,7\end{array}$ & $\begin{array}{l}1,358 \\
51,3\end{array}$ & $\begin{array}{l}1,644 \\
62,1\end{array}$ & $\begin{array}{c}0,166 \\
6,3\end{array}$ & $\begin{array}{l}0,282 \\
10,6\end{array}$ & $\begin{array}{c}0,201 \\
7,6\end{array}$ & $\begin{array}{l}0,445 \\
16,8\end{array}$ \\
\hline
\end{tabular}

Formazan-Methode (Triphenyltetrazoliumchlorid als Reagens) und folgten dabei dem Vorgehen von FaIrbridge und Mitarbeitern (11). $3 \mathrm{~m} l$ des Bebrütungsansatzes wurden mit $1 \mathrm{~m} l$ 20 proz. Trichloressigsäure enteiweißt. $3 \mathrm{ml}$ des Filtrats wurden dann zum Farbreaktionsgemisch gegeben, dessen Rotfärbung mit dem Havemann-Kolorimeter "MGF“ gemessen wurde. Bei der Bestimmung der elektrophoretisch behandelten Enzymproben wurde $1 \mathrm{~cm}$ Schichtdicke gewählt, während die sonstigen Ansätze in der $0,2 \mathrm{~cm}$-Küvette gemessen wurden.

\section{Trägerelektrophorese}

Die Elektrophoreseversuche wurden an einer selbst gebauten Apparatur im Prinzip nach den Angaben von GrassmanN und HaNNIG (12) durchgeführt. Als Träger verwendeten wir vergleichsweise Filterpapier Nr. 2043a Mgl der Fa. Schleicher \& Schüll und Acetylcellulose-Streifen in Form der sog. Membranfolien der Membranfilter-Gesellschaft. In einem Teil der Versuche wurde auch das Papier „Elphor" des VEB Spezialfilterpapierfabrik, verwendet. Auf die puffergetränkten Streifen wurden 20 bzw. $50 \mu l$ Enzymlösung aufgetragen und über $71 / 2$ und $15 \mathrm{Stdn}$. eine Gleichspannung von 110 bzw. $165 \mathrm{~V}$ angelegt. Die Versuche wurden ohne unmittelbare Kühlung des Streifens, jedoch bei verschiedener Raumtemperatur $\left(20^{\circ}, 4^{\circ}, 2^{\circ}\right)$ durchgeführt. Nach beendeter Elektrophorese wurden für die Bebrütungsansätze nach $7 \frac{1}{2} \mathrm{stdg}$. Elektrophorese $10 \mathrm{~cm}$ lange Rumpfstücke und nach $15 \mathrm{stdg}$. Elektrophorese $14 \mathrm{~cm}$ lange Mittelabschnitte des Streifens verwendet, die in jedem Fall die während der Elektrophorese durchlaufende Wegstrecke des Enzyms umfaßten. Die noch feuchten Streifen wurden fein zerschnitten und zum Puffer bzw. zum Substrat-Puffer-Gemisch gegeben.

Um die allein durch die Gegenwart von Papier bzw. Acetylcellulose eintretenden Aktivitätsverluste von den durch den Elektrophoresevorgang selbst bedingten abgrenzen zu können, wurden vergleichende Versuche angestellt; in denen das Enzym auf die puffergetränkten Streifen nur aufgetragen, dann in der angegebenen Weise wieder eluiert wurde.

\section{Ergebnisse}

\section{Amylase}

Die Tabelle 1 ermöglicht den Vergleich der verbleibenden Restaktivitäten auf Papier einerseits und Acetylcellulose andererseits. Mit dem letzteren wird durchweg eine höhere, teilweise bis doppelt so große Enzymaktivität wiedergefunden.

Wir prüften weiterhin, in welchem Maße bereits die vielstündige Verweildauer der Amylase auf dem feuchten Streifen obne Einwirkung des elektrischen Stromes partielle Inaktivierung herbeiführt. Diese Versuche wurden teilweise unter Calciumzusatz durchgeführt, der, wie aus Tabelle 2 hervorgeht, vor allem bei Aufbe-

Tab. 2

Amylase-Aktivität bei Aufbewahren der mit Enzym versehenen Streifen in der feuchten Kammer bei $20^{\circ}$ und bei $2^{\circ}$ (Werte in mg freigesetzte Maltose)

\begin{tabular}{|c|c|c|c|c|c|c|}
\hline Träger & $\begin{array}{l}\text { Sofort in } \\
\text { ohne } \mathrm{Ca}^{1} \text { ) }\end{array}$ & $\begin{array}{l}\text { kubiert } \\
\text { mit } \mathrm{Ca}_{2}\end{array}$ & $\begin{array}{l}\text { Nach } 7 \\
\text { ohne Ca }\end{array}$ & $\begin{array}{l}1 / 2 \text { stdg. } \\
20^{\circ} \mathrm{C} \\
\text { mit } \mathrm{Ca}\end{array}$ & $\begin{array}{r}\text { Auf bew } \\
2^{\circ} \\
\text { a ohne } \mathrm{Ca}\end{array}$ & $\begin{array}{l}\text { vahrung } \\
\text { C mit } \mathrm{Ca}\end{array}$ \\
\hline Papier & 1,619 & 1,625 & 1,221 & 1,366 & 1,587 & 1,628 \\
\hline Acetylcellulose & 2,294 & 2,319 & 1,931 & 2,298 & 2,314 & 2,318 \\
\hline
\end{tabular}

1) Der Calciumzusatz $\left(6,67 \cdot 10^{-3} m \mathrm{CaCl}_{2}\right)$ bezieht sich auf den zum Anfeuchten der Streifen benutzten Acetatpuffer.

wahrung der amylasehaltigen Streifen bei Zimmertemperatur eine stabilisierende Wirkung entfaltet. Auch hier zeitigte Acetylcellulose wiederum weit bessere Enzymausbauten als Papier. - Úbrigens war Calcium, dem Elektrophoresepuffer zugesetzt, nicht in der Lage, die in Tabelle 1 aufgezeigten bei der Elektrophorese eintretenden Aktivitätsverluste auch nur geringfügig herabzusetzen. Dagegen steigerte Zusatz von Äthylendiamintetraacetat (EDTA) in allen Versuchen die Amylaseaktivität, wobei der Komplexbildner teils zur Inkubationsflüssigkeit, teils zum Elektrophoresepuffer gegeben wurde, wie Tabelle 3 zeigt. - Mit Acetylcellulose wurden prinzipiell die gleichen Befunde wie mit dem mit Enzym versehenen Papier erhoben, nur daß eben bei dem ersteren durchweg weit höhere Aktivitäts-

Tab. 3. Einfluß von Athylendiamintetraacetat auf die Amylase-Aktivität vor und nach Trägerelektrophorese (Werte in mg freigesetzte Máltose bzw. \% Aktivität)

\begin{tabular}{|c|c|c|c|c|c|c|c|c|}
\hline \multirow[t]{3}{*}{ Träger } & \multicolumn{4}{|c|}{ ohne Träger Enzymlösung } & \multicolumn{4}{|c|}{$\begin{array}{c}\text { Nach 71/2stdg. Elektrophorese } \\
\text { Elektrophoresepuffer }\end{array}$} \\
\hline & \multicolumn{2}{|c|}{ Inkubation } & \multicolumn{2}{|c|}{ Inkubation } & \multirow{2}{*}{\multicolumn{2}{|c|}{$\begin{array}{l}\text { ohne EDTA Inkubation } \\
\text { ohne EDTA mit EDTA }\end{array}$}} & \multirow{2}{*}{\multicolumn{2}{|c|}{$\begin{array}{l}\text { mit EDTA Inkubation } \\
\text { ohne EDTA mit EDTA }\end{array}$}} \\
\hline & ohne EDTA & mit $^{1}$ ) EDTA & ohne EDTA & mit EDTA & & & & \\
\hline Papier & $\begin{array}{c}2,788 \\
100\end{array}$ & $\begin{array}{l}2,834 \\
(100)\end{array}$ & $\begin{array}{l}1,706 \\
61,2\end{array}$ & $\begin{array}{l}1,931 \\
(68,1)\end{array}$ & $\begin{array}{c}0,640 \\
23,0\end{array}$ & $\begin{array}{l}0,853 \\
(30,1)\end{array}$ & $\begin{array}{l}0,935 \\
33,5\end{array}$ & $\begin{array}{l}1,087 \\
(38,4)\end{array}$ \\
\hline Acetylcellulose & - & - & $\begin{array}{c}2,334 \\
83,7\end{array}$ & $\begin{array}{l}2,497 \\
(88,1)\end{array}$ & $\begin{array}{l}1,390 \\
50,0\end{array}$ & $\begin{array}{l}1,420 \\
(50,1)\end{array}$ & $\begin{array}{l}1,400 \\
50,2\end{array}$ & $\begin{array}{l}1,974 \\
(69,6)\end{array}$ \\
\hline
\end{tabular}

1) $6,67 \cdot 10^{-3} m \mathrm{EDTA}$. 
werte resultierten. Diese betrugen in den Elektrophoreseversuchen etwa $150-200 \%$ der enzymatischen Aktivität, die bei Verwendung von Papier als Träger gemessen wurde.

\section{$\beta$-Glucuronidase}

Auch bei diesem Enzym bewährte sich Acetylcellulose als Träger weit besser als Papier, wie Tabelle 4 zeigt. In diese Versuche wurde auch das Papier „Elphor" mit einbezogen; es hatte sich in vergleichenden Untersuchungen über den Einfluß der Papiersorte auf die Aktivität verschiedener Enzyme unter sieben Papieren neben $2043 \mathrm{a}$ als besonders günstig erwiesen.

Tab. 4

$\beta$-Glucuronidase-Aktivität vor und nach Elektrophorese auf Papier bzw. Acetylcellulose (15 Stdn.; $165 \mathrm{~V} ; 4^{\circ}$ )

\begin{tabular}{|c|c|c|c|c|c|c|}
\hline \multirow[t]{2}{*}{ Träger } & $\begin{array}{l}\text { Gluc- } \\
\text { uron- } \\
\text { säure }\end{array}$ & $\begin{array}{l}\text { Akti- } \\
\text { vität }\end{array}$ & $\begin{array}{l}\text { Gluc- } \\
\text { uron- } \\
\text { säure }\end{array}$ & $\begin{array}{r}\text { Akti- } \\
\text { vität }\end{array}$ & $\begin{array}{c}\text { Nach } \\
\text { troph } \\
\text { Gluc- } \\
\text { uron- } \\
\text { säure }\end{array}$ & $\begin{array}{c}\text { Elek- } \\
\text { Lorese } \\
\text { Akti- } \\
\text { vität }\end{array}$ \\
\hline & $\mathrm{mg}$ & $\%$ & $\mathrm{mg}$ & $\%$ & $\mathrm{mg}$ & $\%$ \\
\hline Papier 2043a & 0,490 & 100 & 0,330 & 67,4 & 0,120 & 24,5 \\
\hline Papier „Elphor“ & 0,490 & 100 & 0,275 & 56,1 & 0,100 & 20,4 \\
\hline Acetylcellulose & 0,440 & 100 & 0,425 & 96,1 & 0,355 & 80,7 \\
\hline
\end{tabular}

\section{Diskussion}

Aus allen Versuchen geht hervor, daß Acetylcellulose selbst bestem Filterpapier hinsichtlich der Erhaltung der Enzymaktivität überlegen ist. Dieser günstige Effekt wird in den Elektrophorese-Experimenten besonders deutlich. Da hierbei das Enzym mit dem Träger in besonders innigen Kontakt kommt und schließlich auf größere Oberfläche ausgebreitet wird als beim bloßen Auftragen auf den Streifen, müssen sich bei diesem Verfahren Adsorptionserscheinungen zwangsläufig in erhöhtem Maße äußern. Von Acetylcellulose aber ist bekannt, daß sie Proteine nicht oder nur in sehr geringem Umfang adsorbiert (19-21). Dies dürfte auch der hauptsächliche Grund für die höhere Enzymaktivität bei Verwendung von Acetylcellulose in der Trägerelektrophorese sein. Die Enzymadsorption wird durch die Gegenwart polyvalenter Anionen herabgesetzt (13). Der dementsprechend von Mrchl (14) vorgeschlagene Zusatz eines Proteins zum Puffer hat sich auch in unserem Laboratorium z. B. bei der Papierelektrophorese von Hyaluronidase bewährt (15). Er ist beim Arbeiten mit Enzymen jedenfalls dem von anderer Seite zwecks Herabsetzung der Proteinadsorption empfohlenen Imprägnieren des Papiers mit einem Detergens $(16,17)$ vorzuziehen. Aus dem Gesagten läßt sich ableiten, daß die durch Adsorption bedingten Enzymverluste um so größer sein werden, je reiner das der Papierelektrophorese unterworfene Enzympräparat ist. Wir haben das an verschiedenen kristallisierten Enzymen demonstrieren können (18). Wie eingangs angedeutet, ist auch eine Schädigung von Enzymen durch Inhaltsstoffe des Papiers in Betracht zu ziehen. Nach unseren Untersuchungen (3) kommt hierbei dem Schwermetallgehalt (Fe!) - auch der in der Papierchromatographie und -elektrophorese bewährten Papiersorten - besondere Bedeutung $z u$, vor allem bei der Elektrophorese SH-abhängiger Enzyme. Acetylcellulose ist aber in größerer chemischer Reinheit erhältlich als Papier. - $\mathrm{Da} \beta$ auch bei der Trägerelektrophorẹse mit Acetylcellulose Aktivitätsverluste von Enzymen auftreten, ist natürlich auf Denaturierung zurückzuführen, die ohne zusätzliche Kühlung am Träger kaum völlig auszuschließen ist. Immerhin scheint uns das genannte Verfahren empfehlenswert, wenn es darauf ankommt, ohne großen Aufwand Enzyme elektrophoretisch zu behandeln. Im Hinblick auf den relativ hohen Preis derartiger Actylcellulosefolien ist es von Vorteil, daß ihre Wiederverwendung z. B. nach der Elektrophorese von Serumproteinen möglich ist (22).

\section{Literatur}

1. Methfessel, J., Naturwissenschaften 44, 329 (1957). - 2. MethFESSEL, J., IV. Internat. Kongreß für Biochemie, Zusammenfassungen 4 -118, Wien (1958). - 3. Methfesser, J., Wiss. Z. Martin-Luther-Univ. Halle-Wittenberg, math.-naturwiss. R. XII/2, 123 (1963). - 4. Gordon, A. H., B. Keil und K. Sebesta, Nature (London) 164, 498 (1949). - 5. Smithies, O., Biochem. J. 61, 629 (1955). - 6. Dose, K. und G. Krause, Naturwissenschaften 49, 349 (1962). - 7. Noelting, G. und P. BerNFELD, Helv. chim. Acta 31, 286 (1948). - 8. Fishman, W. H., J. biol. Chemistry 127, 367 (1939). - 9. Weichselbaum, T. E., Amer. J. Clin. Path. 10, 40 (1946). - 10. Qurck, A. J., J. biol. Chemistry 61, 667 (1924). - 11. FAIRbridge, R. A., K. J. Willis und R. G. Bоoth, Biochem. J. 49, 423 (1951). - 12. GrassmanN,
W. und K. Hannig, Hoppe-Seyler's Z. physiol. Chem. 290, 1 (1952). - 13. Colowick, S. P. in Colowick-Kaplan, Methods in Enzymology I, S. 90, Acad. Press Inc., New York (1955). 14. Mrchi, H., Mh. Chem. 85, 1251 (1954). - 15. Nrurus, R., Dissertation, Med. Fak. Halle (1961). - 16. Trselius, A. und P. Flodin, Advances Protein Chemistry VIII, S. 461, Acad. Press. Inc., New Yotk (1956). - 17. Monty, K. J., M. Morrison, E. Alling und E. Storz, J. biol. Chemistry 220, 295 (1956). 18. Methresser, J., Habilitationsschrift Halle (1960). - 19. KohN, J., Nature (London) 181, 839 (1958). - 20. Pieper, J., Zschr. exper. Med. 131, 359 (1959). - 21. WirLand, Th., G. Pfleiderer, J. Haupt und W. Wörnèr, Biochem. Z. 332, 1 (1959). - 22. JACOBS, S., Nature (London) 183, 1326 (1959). 